\title{
Quelques et plusieurs ${ }^{1}$
}

\author{
Philippe Gréa \\ MoDyCo - Université Paris 10 \& CNRS \\ philippe.grea@u-paris10.fr
}

\section{Orientation argumentative et petite quantité}

\subsection{Une orientation argumentative inversée ?}

Depuis l'article de Gondret (1976), l'un des facteurs communément avancé pour distinguer quelques et plusieurs tient dans le fait que quelques, contrairement à plusieurs, s'accommode facilement de contextes minorants $^{2}$ :

1. a. Il ne possède que \{quelques / *plusieurs\} livres.

b. Il possède seulement \{quelques / *plusieurs\} livres.

c. Je n'ai lu que \{quelques / *plusieurs\} livres.

d. \{quelques / *plusieurs\} personnes seulement assistaient à la réunion.

e. $\{$ quelques / *plusieurs\} livres me suffisent.

f. Personne n'y croyait sauf \{quelques / *plusieurs\} inconditionnels.

Cette explication est officialisée dans la Grammaire méthodique du français qui traduit cette opposition en termes d'échelles argumentatives. Cette thèse est reprise et développée par Bacha (1997). Selon elle, choisir quelques ou plusieurs, c'est orienter l'interprétation respectivement vers le négatif ou le positif. Dans les exemples qui suivent, elle note ainsi que quelques impose un aspect restrictif alors que plusieurs implique au contraire une augmentation :

2. a. Ne nous affolons pas : nous sommes encore à \{plusieurs /? quelques\} semaines des élections.

b. Mon dieu que le temps passe vite, nous sommes déjà à \{?plusieurs / quelques\} semaines des élections.

c. Tu as manqué le train de peu : il est parti il y a \{?plusieurs / quelques\} minutes.

d. Tu es vraiment en retard : le train est parti il y a \{plusieurs / ? quelques\} minutes.

Plus récemment, Leeman (2004) reconduit cette analyse et dans un tout autre cadre théorique, Jayez (2005) avance une formalisation des propositions de Bacha. La valeur restrictive de quelques et augmentative de plusieurs fait donc aujourd'hui plus ou moins consensus.

Pourtant, cette analyse, qui se réclame des travaux de Ducrot et d'Anscombre sur l'argumentation, est discutable. En effet, Ducrot (1980) montre qu'une phrase comme Il a lu quelques romans de Balzac est orientée vers une conclusion positive (contrairement à la phrase il n'a pas lu tous les romans de Balzac qui est orientée vers une conclusion négative du type : la personne en question connaît mal Balzac). Ce résultat, dont Ducrot souligne lui-même le caractère contre-intuitif, est démontré d'une manière tout à fait classique dans le cadre de la théorie de l'argumentation. Il suffit en effet d'observer que les deux énoncés n'appellent pas le même type de conclusion :

3. a. Il n'a pas lu tous les romans de Balzac. *Il te donnera peut-être le renseignement que tu désires.

b. Il a lu quelques romans de Balzac. Il te donnera peut-être le renseignement que tu désires.

Si dans 3a, l'enchaînement n'est pas permis, alors qu'il l'est dans 3b, c'est que les deux phrases ont une orientation argumentative différente. L'orientation positive de quelques ne fait pas non plus de doute dans les exemples suivants, comme le note fort bien Gaatone (1991) et Gaatone (2006) :

4. a. J'ai quelques / plusieurs amis *et même pas du tout.

b. J'ai quelques / ?plusieurs amis et même beaucoup. 
c. Heureusement, quelques / plusieurs amis sont venus m'aider.

d. *Malheureusement, quelques / plusieurs amis sont venus m'aider.

Dans 4a, il est impossible d'enchaîner avec la conclusion et même pas du tout, d'orientation négative. Au contraire, la conclusion et même beaucoup, d'orientation positive, ne pose aucun problème. On constate aussi que dans 4c, l'adverbe de phrase heureusement a la même orientation (positive) que le reste de la phrase. Si quelques avait une orientation négative, on ne comprendrait pas le caractère naturel de cet enchaînement.

Par conséquent, aucun élément ne permet de dire que quelques et plusieurs ont une orientation argumentative opposée. La seule chose que montrent les exemples 1 et 2, c'est que quelques n'est pas incompatible avec les contextes restrictifs.

\subsection{Une quantité plus ou moins faible ?}

Le second point traditionnellement discuté lorsqu'on compare quelques et plusieurs concerne la plus ou moins grande quantité exprimée par les deux déterminants. Habituellement, on s'accorde à dire que cette quantité est peu élevée et certains auteurs ne craignent pas d'aller plus loin en fixant un intervalle. Ainsi, en se fondant sur l'exemple suivant :

5. Cette semaine nous avons planté plusieurs / quelques arbres dont $5 / 10 /$ ?15 /??30 ont dî être arrachés.

Asnès (2006) est amenée à conclure :

«On constate à partir de l'exemple 5 que moins grande est la cardinalité dénotée par plusieurs $\mathrm{N}$ et quelques $\mathrm{N}$, plus acceptable est la phrase. On constate que la borne supérieure imposée par plusieurs / quelques se situe autour du nombre 10. » Asnès (2006, p. 339)

Mais ce n'est pourtant pas ce qui vient à l'esprit des auditeurs quand ils entendent à la radio : quelques gouttes affecteront la pointe du Finistère. Car en admettant que la pointe du Finistère fasse $6400 \mathrm{Km}^{2}$, le bulletin météo donnerait, selon Asnès, un maximum d'une goutte tous les $640 \mathrm{Km}^{2}$ ou encore $1,56 * 10^{-9}$ goutte par $\mathrm{m}^{2}$, ce qui semble assez éloigné de ce que veut effectivement signifier l'annonce radiophonique.

Les avis divergent lorsqu'il s'agit d'ordonner les deux déterminants entre eux et de préciser cette petite quantité. Gondret, qui défend le caractère augmentatif de plusieurs et diminutif de quelques, en vient ainsi à inverser l'ordre habituellement présenté dans les dictionnaires et les grammaires : "On pourrait presque changer l'ordre des termes de la Grammaire Larousse et dire que dans le discours, souvent, plusieurs s'applique à une quantité plus petite que quelques. » Gondret (1976, p. 148).

La proposition inverse est aussi avancée en s'appuyant sur un test qui présente, au-delà des auteurs qui y ont recours, le même mode opératoire. Par exemple, Bacha (1997) et Leeman (2004, p.165) posent à des locuteurs une question du genre : «J'ai invité 20 personnes, quelques-unes sont venues : combien y avaitil de présents ? » Ou encore, « J'ai invité 20 personnes, plusieurs ne sont pas venues : combien à peu près selon vous ? ». Les résultats de cette enquête sont plutôt opposés à ceux de Gondret : «...le petit écart, ..., tendrait à faire penser que plusieurs évoque une quantité légèrement plus importante que quelques » Leeman (2004, p. 166). On retrouve une expérience analogue dans Spector (2006, p.244) où l'on demande à des sujets d'évaluer la vérité des phrases 6 relativement à des feuilles de papier sur lesquelles figurent un, deux, trois, ou plus de trois points.

6. a. Il y a quelques points sur la feuille.

b. Il y a plusieurs points sur la feuille.

Il est vrai que l'objectif de cette dernière enquête est un peu différent de ce qui précède, puisqu'il s'agit de montrer le caractère vague du déterminant quelques (contrairement à plusieurs), en constatant que les 
sujets sont généralement embarrassés et hésitent à valider la phrase 6a quand il y a seulement deux points sur la feuille.

De telles expériences, selon nous, donnent des résultats contradictoires. Elles se fondent en effet sur une question mal posée (quelle est la quantité dénotée par quelques et plusieurs?) qui ne peut apporter que de mauvaises réponses.

\section{Méthodologie}

Un certain nombre de faits, que nous allons présenter dans ce qui suit, nous amènent en effet à penser que cette différence de sens, que tout le monde observe entre quelques et plusieurs, gagnerait à être traitée dans les termes d'une différence de sommation qui, en dernier lieu, est susceptible d'être représentée dans le cadre de la Grammaire cognitive de Langacker (1987) et Langacker (1991). Pour appuyer notre raisonnement, nous avons utilisé la base Frantext catégorisée en élaborant un protocole d'extraction de données que nous décrivons dans cette section.

Une première étape a consisté à extraire l'ensemble des syntagmes [quelques $N$ ] (63098 occurrences, corpus Q) et [plusieurs N] (21009 occurrences, corpus P), soit un corpus total (désormais noté C) de 84107 phrases $^{3}$. Pour les deux sous-corpus, Q et $\mathrm{P}$, nous avons généré les index de fréquences des $\mathrm{N}$ qui suivent les deux déterminants. A titre d'illustration, voici la liste des dix substantifs les plus fréquents après quelques et plusieurs :

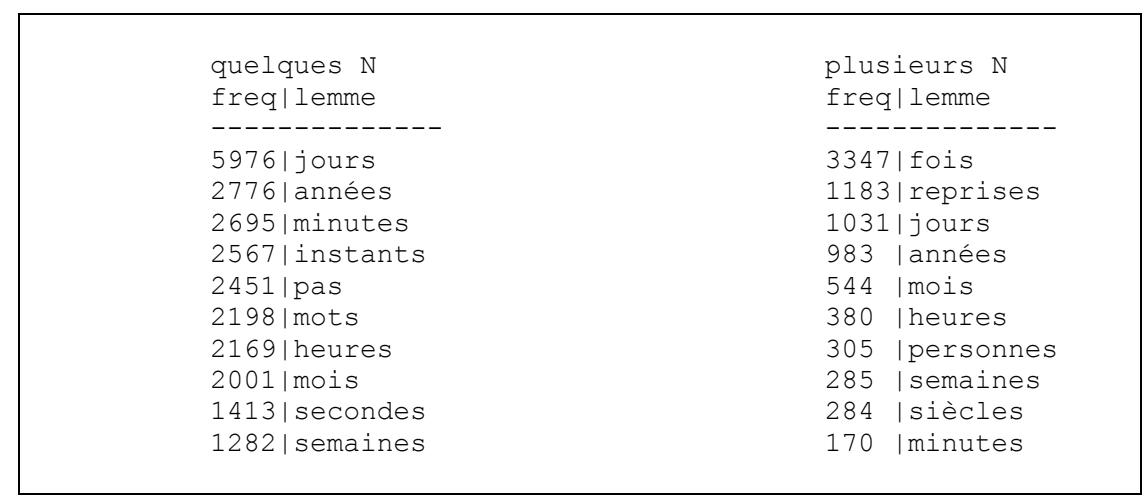

Tableau $1:$ index de fréquences des $\mathrm{N}$ après quelques et plusieurs ${ }^{4}$

On constate que beaucoup des termes fréquents apparaissent dans les deux listes (par ex. jours, années, heures, mois, semaines, etc.). Ils ne sont donc pas nécessairement spécifiques d'un déterminant et n'apportent aucune information pertinente sur ce qui permettrait de les distinguer.

Il existe cependant plusieurs méthodes permettant de calculer la surreprésentation d'une forme dans un sous-corpus (ici, Q ou P) par rapport à un corpus de référence (ici, C). L'une d'entre elles repose sur une loi probabiliste, la loi hypergéométrique, qui permet dans le cas qui nous intéresse de mesurer la spécificité d'un substantif pour un déterminant donné, c'est-à-dire la surreprésentation de ce substantif avec ce déterminant par rapport à une distribution aléatoire ou « régulière » entre les deux déterminants ${ }^{5}$.

Cette mesure se présente, dans le tableau qui suit, sous la forme d'une répartition des substantifs entre 1 et $0^{6}$. Les valeurs proches de (ou égales à) 1 (notées dans la colonne taux) indiquent que les substantifs (dans la colonne lemme) sont des spécificités positives de quelques, c'est-à-dire sont surreprésentés dans le sous-corpus Q. 


\begin{tabular}{|c|c|c|c|c|c|c|c|c|}
\hline \multicolumn{8}{|c|}{ quelques $\mathrm{N}$} & subfreq \\
\hline 1 & I I & vers & । & 1.00000 & 1 & 220 & 1 & 218 \\
\hline 2 & 1 & sous & । & 1.00000 & i & 249 & i & 243 \\
\hline 3 & 1 & secondes & । & 1.00000 & । & 1451 & I & 1413 \\
\hline 4 & i & phrases & | & 1.00000 & । & 372 & i & 354 \\
\hline 5 & $1 \mathrm{r}$ & pas & | & 1.00000 & 1 & 2468 & 1 & 2451 \\
\hline 6 & $1 \mathrm{r}$ & paroles & । & 1.00000 & । & 471 & 1 & 469 \\
\hline 7 & $1 \mathrm{r}$ & pages & 1 & 1.00000 & | & 599 & i & 547 \\
\hline 8 & $1 \mathrm{n}$ & mètres & । & 1.00000 & । & 543 & I & 491 \\
\hline 9 & in & mots & | & 1.00000 & | & 2229 & i & 2198 \\
\hline 10 & $\mathrm{n}$ & moments & | & 1.00000 & | & 248 & i & 244 \\
\hline 11 & I $n$ & minutes & | & 1.00000 & | & 2865 & 1 & 2695 \\
\hline 12 & 11 & lignes & | & 1.00000 & | & 791 & 1 & 754 \\
\hline 13 & 1 & jours & । & 1.00000 & । & 7007 & । & 5976 \\
\hline 14 & 1 & instants & | & 1.00000 & i & 2570 & i & 2567 \\
\hline 15 & $1 \mathrm{r}$ & heures & । & 1.00000 & । & 2549 & । & 2169 \\
\hline 16 & I & gouttes & | & 1.00000 & । & 450 & i & 449 \\
\hline 17 & 10 & détails & 1 & 1.00000 & । & 313 & i & 292 \\
\hline 18 & 1 & larmes & 1 & 0.99999 & । & 143 & 1 & 142 \\
\hline 19 & 1 & amis & | & 0.99999 & । & 360 & 1 & 329 \\
\hline 20 & i r & notes & i & 0.99999 & । & 180 & i & 174 \\
\hline 21 & $1 \epsilon$ & exceptions & । & 0.99999 & । & 113 & । & 113 \\
\hline 22 & 1 & fleurs & । & 0.99999 & i & 131 & i & 128 \\
\hline 23 & 1 & renseignements & 1 & 0.99999 & 1 & 109 & 1 & 108 \\
\hline 24 & 1 & indications & | & 0.99999 & । & 132 & 1 & 128 \\
\hline 25 & 1 & semaines & । & 0.99999 & । & 1567 & i & 1282 \\
\hline 26 & I & précautions & । & 0.99999 & । & 82 & । & 82 \\
\hline 27 & $1+$ & traits & । & 0.99999 & । & 250 & i & 227 \\
\hline 28 & 1 & réflexions & । & 0.99999 & । & 96 & । & 94 \\
\hline 29 & 1 & grains & | & 0.99999 & । & 67 & i & 67 \\
\hline 30 & $1+$ & traces & । & 0.99999 & । & 78 & i & 77 \\
\hline 31 & I & centimètres & । & 0.99999 & । & 204 & । & 185 \\
\hline 32 & I $n$ & marches & । & 0.99999 & I & 203 & I & 184 \\
\hline 33 & 13 & bribes & i & 0.99999 & i & 60 & i & 60 \\
\hline 34 & 1 & souvenirs & | & 0.99999 & । & 78 & i & 76 \\
\hline 35 & i & arbres & | & 0.99999 & i & 136 & i & 126 \\
\hline 36 & 1 & idées & | & 0.99999 & | & 177 & 1 & 160 \\
\hline 37 & $1 \epsilon$ & esprits & । & 0.99999 & । & 117 & I & 109 \\
\hline 38 & 1 & exemples & | & 0.99999 & i & 314 & i & 271 \\
\hline 39 & 11 & leçons & | & 0.99999 & | & 79 & 1 & 76 \\
\hline 40 & $1 \mathrm{r}$ & nuages & । & 0.99999 & । & 51 & । & 51 \\
\hline 41 & 1 & lueurs & | & 0.99999 & | & 50 & i & 50 \\
\hline 42 & I & débris & | & 0.99999 & । & 69 & I & 67 \\
\hline 43 & I & précisions & | & 0.99999 & i & 60 & i & 59 \\
\hline 44 & 11 & lumières & | & 0.99999 & | & 49 & 1 & 49 \\
\hline 45 & I r & notions & । & 0.99999 & । & 84 & I & 80 \\
\hline 46 & I & cheveux & | & 0.99999 & I & 58 & I & 57 \\
\hline 47 & $1 \mathrm{k}$ & propos & | & 0.99999 & | & 66 & | & 64 \\
\hline 48 & I & mois & | & 0.99999 & i & 2545 & i & 2001 \\
\hline 49 & 1 & louis & | & 0.99999 & | & 45 & | & 45 \\
\hline 50 & $1 \mathrm{R}$ & poignées & । & 0.99999 & 1 & 55 & i & 54 \\
\hline 51 & 1 & francs & । & 0.99999 & । & 44 & । & 44 \\
\hline 52 & 1 & livres & | & 0.99999 & i & 234 & i & 203 \\
\hline 53 & 1 & fruits & | & 0.99999 & I & 53 & i & 52 \\
\hline 54 & 1 & regards & | & 0.99999 & | & 43 & i & 43 \\
\hline 55 & 1 & gestes & | & 0.99999 & । & 43 & । & 43 \\
\hline 56 & 1 & doutes & | & 0.99999 & i & 43 & i & 43 \\
\hline 57 & $1 \epsilon$ & écus & । & 0.99999 & I & 42 & I & 42 \\
\hline 58 & 1 & réserves & । & 0.99998 & । & 40 & I & 40 \\
\hline 59 & $1 \epsilon$ & efforts & । & 0.99998 & । & 57 & I & 55 \\
\hline 60 & I & poils & । & 0.99998 & । & 38 & I & 38 \\
\hline 61 & 1 & dixièmes & | & 0.99997 & i & 37 & | & 37 \\
\hline 62 & 1 & gens & | & 0.99996 & | & 61 & | & 58 \\
\hline
\end{tabular}




\begin{tabular}{|c|c|c|c|c|c|c|c|}
\hline 63 & 1 & hommes & I & 0.99996 & 1 & 599 & 488 \\
\hline 64 & I & chiffres & I & 0.99996 & I & 74 & 69 \\
\hline 65 & 1 & lambeaux & । & 0.99995 & I & 44 & 43 \\
\hline 66 & i & difficultés & i & 0.99994 & i & 85 & 78 \\
\hline 67 & । & mesures & । & 0.99994 & | & 91 & 83 \\
\hline 68 & i & progrès & i & 0.99994 & i & 34 & 34 \\
\hline 69 & i & taches & I & 0.99993 & | & 43 & 42 \\
\hline 70 & 1 & restes & । & 0.99993 & I & 43 & 42 \\
\hline 71 & i & gorgées & i & 0.99993 & i & 58 & 55 \\
\hline 72 & । & observations & । & 0.99992 & | & 124 & 110 \\
\hline 73 & i & chaises & i & 0.99990 & i & 70 & 65 \\
\hline 74 & 1 & oiseaux & । & 0.99990 & | & 49 & 47 \\
\hline 75 & i & provisions & । & 0.99989 & i & 32 & 32 \\
\hline 76 & 1 & privilégiés & । & 0.99989 & i & 32 & 32 \\
\hline 77 & I & coups & I & 0.99988 & I & 345 & 286 \\
\hline 78 & । & brins & । & 0.99987 & I & 48 & 46 \\
\hline 79 & i & objections & i & 0.99986 & i & 55 & 52 \\
\hline 80 & 1 & vestiges & । & 0.99986 & i & 31 & 31 \\
\hline 81 & i & meubles & i & 0.99979 & i & 53 & 50 \\
\hline 82 & i & éclats & । & 0.99976 & | & 29 & 29 \\
\hline 83 & i & miettes & i & 0.99976 & i & 29 & 29 \\
\hline 84 & 1 & billets & i & 0.99966 & i & 104 & 92 \\
\hline 85 & i & recherches & I & 0.99957 & | & 27 & 27 \\
\hline 86 & i & différences & । & 0.99957 & I & 27 & 27 \\
\hline 87 & 1 & plaisanteries & । & 0.99950 & | & 35 & 34 \\
\hline 88 & । & soupirs & । & 0.99943 & | & 26 & 26 \\
\hline 89 & 1 & sacrifices & । & 0.99943 & i & 26 & 26 \\
\hline 90 & I & légumes & । & 0.99943 & | & 26 & 26 \\
\hline 91 & । & enjambées & । & 0.99943 & | & 26 & 26 \\
\hline 92 & i & compliments & i & 0.99943 & i & 26 & 26 \\
\hline 93 & i & bouchées & i & 0.99935 & i & 34 & 33 \\
\hline 94 & 1 & bonds & । & 0.99935 & i & 34 & 33 \\
\hline 95 & i & chances & । & 0.99930 & I & 41 & 39 \\
\hline 96 & i & éclaircissements & 1 & 0.99924 & | & 25 & 25 \\
\hline 97 & i & soins & i & 0.99924 & i & 25 & 25 \\
\hline 98 & । & instans & । & 0.99924 & | & 25 & 25 \\
\hline 99 & । & pièces & । & 0.99916 & | & 310 & 254 \\
\hline 100 & i & expressions & । & 0.99912 & | & 40 & 38 \\
\hline 101 & । & ressources & । & 0.99899 & | & 24 & 24 \\
\hline 102 & i & lecteurs & i & 0.99899 & i & 24 & 24 \\
\hline 103 & । & vieillards & । & 0.99898 & | & 46 & 43 \\
\hline 104 & । & papiers & । & 0.99873 & | & 63 & 57 \\
\hline 105 & I & arpents & । & 0.99859 & | & 38 & 36 \\
\hline 106 & 1 & explications & । & 0.99855 & i & 100 & 87 \\
\hline 107 & i & pieds & i & 0.99851 & i & 135 & 115 \\
\hline 108 & । & étoiles & । & 0.99847 & | & 62 & 56 \\
\hline 109 & I & avances & । & 0.99820 & I & 22 & 22 \\
\hline 110 & i & pierres & । & 0.99816 & | & 61 & 55 \\
\hline 111 & i & mèches & । & 0.99776 & | & 36 & 34 \\
\hline 112 & । & bijoux & । & 0.99776 & | & 36 & 34 \\
\hline 113 & । & roses & । & 0.99760 & | & 21 & 21 \\
\hline 114 & i & connaissances & । & 0.99757 & | & 42 & 39 \\
\hline 115 & 1 & pensées & । & 0.99756 & I & 48 & 44 \\
\hline 116 & i & trucs & i & 0.99681 & i & 20 & 20 \\
\hline 117 & I & pommes & । & 0.99681 & | & 20 & 20 \\
\hline 118 & I & conseils & I & 0.99668 & | & 79 & 69 \\
\hline 119 & 1 & fragments & । & 0.99663 & | & 109 & 93 \\
\hline 120 & i & regrets & i & 0.99616 & I & 27 & 26 \\
\hline 121 & I & parcelles & । & 0.99616 & | & 27 & 26 \\
\hline 122 & I & intimes & I & 0.99616 & I & 27 & 26 \\
\hline 123 & I & rires & I & 0.99575 & I & 19 & 19 \\
\hline 124 & I & pointes & । & 0.99575 & I & 19 & 19 \\
\hline 125 & I & oliviers & । & 0.99575 & | & 19 & 19 \\
\hline 126 & I & cuillerées & I & 0.99575 & I & 19 & 19 \\
\hline 127 & I & éclairs & I & 0.99508 & I & 26 & 25 \\
\hline 128 & i & défauts & I & 0.99508 & I & 26 & 25 \\
\hline 129 & 1 & bruits & | & 0.99508 & | & 26 & 25 \\
\hline
\end{tabular}




\begin{tabular}{|l|l|r|r|r|}
\hline 130 & bouquets & 0.99508 & 26 & 25 \\
131 & pouces & 0.99463 & 44 & 40 \\
132 & hésitations & 0.99443 & 32 & 30 \\
133 & passants & 0.99437 & 38 & 35 \\
134 & étincelles & 0.99433 & 18 & 18 \\
135 & remords & 0.99433 & 18 & 18 \\
136 & pleurs & 0.99433 & 18 & 18 \\
137 & inquiétudes & 0.99433 & 18 & 18 \\
138 & centimes & 0.99433 & 18 & 18 \\
139 & cas & 0.99433 & 264 & 213 \\
140 & remarques & 0.99417 & 95 & 81 \\
141 & principes & 0.99397 & 75 & 65 \\
142 & répliques & 0.99369 & 25 & 24 \\
143 & touffes & 0.99304 & 31 & 29 \\
144 & sylabes & 0.99304 & 31 & 29 \\
145 & ruines & 0.99245 & 17 & 17 \\
146 & illusions & 0.99245 & 17 & 17 \\
147 & espérances & 0.99245 & 17 & 17 \\
148 & droits & 0.99245 & 17 & 17 \\
149 & dettes & 0.99245 & 17 & 17 \\
150 & biscuits & 0.99245 & 17 & 17 \\
& & & & \\
\hline
\end{tabular}

Tableau 2 : N spécifiques de quelques

Prenons le cas de la première ligne, à savoir vers (colonne num, rang numéro 1). Dans le corpus $\mathrm{C}$, vers apparaît en tout (c'est-à-dire, précédé de quelques ou de plusieurs indifféremment) 220 fois (colonne freq). Néanmoins, parmi ces 220 occurrences, on constate que 218 suivent le déterminant quelques (colonne subfreq) et que par conséquent, seules 2 occurrences apparaissent après plusieurs. Nous sommes donc loin d'une situation d'indépendance statistique, dans laquelle les occurrences de vers seraient réparties de manière équilibrée entre les deux sous-corpus (modulo leur taille respective). Cette répartition fait de vers une spécificité positive de quelques.

On trouve la liste des 150 substantifs les plus spécifiques de plusieurs dans le tableau qui suit :

\begin{tabular}{|c|c|c|c|c|c|c|}
\hline \multicolumn{7}{|c|}{ plusieurs N } \\
\hline num & I lemme & । & taux & I & freq & I subfreq \\
\hline--- & -------ー--- & -- & --------- & -- & ----- & ------- \\
\hline 1 & | étages & । & 1.00000 & । & 68 & 63 \\
\hline 2 & | variables & | & 1.00000 & | & 34 & 32 \\
\hline 3 & | systèmes & । & 1.00000 & | & 48 & 41 \\
\hline 4 & I sortes & । & 1.00000 & । & 68 & 68 \\
\hline 5 & | siècles & | & 1.00000 & | & 418 & 284 \\
\hline 6 & | reprises & । & 1.00000 & । & 1185 & 1183 \\
\hline 7 & I personnes & । & 1.00000 & I & 714 & 305 \\
\hline 8 & | millions & | & 1.00000 & i & 212 & 126 \\
\hline 9 & | milliers & । & 1.00000 & I & 392 & 189 \\
\hline 10 & manières & | & 1.00000 & | & 78 & 73 \\
\hline 11 & | langues & । & 1.00000 & i & 74 & 66 \\
\hline 12 & I générations & । & 1.00000 & । & 191 & 164 \\
\hline 13 & groupes & | & 1.00000 & | & 132 & 79 \\
\hline 14 & I fois & I & 1.00000 & । & 3397 & 3347 \\
\hline 15 & f façons & | & 1.00000 & | & 64 & 53 \\
\hline 16 & | espèces & 1 & 1.00000 & । & 108 & 83 \\
\hline 17 & I endroits & । & 1.00000 & । & 175 & 108 \\
\hline 18 & I couches & । & 1.00000 & i & 42 & 38 \\
\hline 19 & | choses & । & 1.00000 & | & 138 & 131 \\
\hline 20 & I causes & । & 1.00000 & i & 53 & 44 \\
\hline 21 & | pays & । & 0.99999 & i & 124 & 74 \\
\hline 22 & I centaines & । & 0.99999 & I & 721 & 276 \\
\hline 23 & I méthodes & । & 0.99999 & । & 31 & 29 \\
\hline 24 & | séries & । & 0.99999 & | & 33 & 30 \\
\hline
\end{tabular}




\begin{tabular}{|c|c|c|c|c|c|c|c|}
\hline 25 & 1 & facteurs & I & 0.99999 & I & 30 & 28 \\
\hline 26 & 1 & niveaux & । & 0.99999 & । & 24 & 24 \\
\hline 27 & | & écoles & 1 & 0.99999 & i & 37 & 32 \\
\hline 28 & | & classes & | & 0.99999 & 1 & 37 & 32 \\
\hline 29 & | & dimensions & | & 0.99999 & i & 29 & 27 \\
\hline 30 & | & nuits & | & 0.99999 & i & 123 & 70 \\
\hline 31 & | & départements & | & 0.99999 & i & 48 & 37 \\
\hline 32 & i & raisons & i & 0.99999 & i & 116 & 67 \\
\hline 33 & 1 & sections & 1 & 0.99999 & i & 35 & 30 \\
\hline 34 & | & sens & | & 0.99999 & i & 32 & 28 \\
\hline 35 & i & catégories & i & 0.99999 & i & 34 & 29 \\
\hline 36 & 1 & solutions & i & 0.99999 & i & 31 & 27 \\
\hline 37 & | & vies & i & 0.99999 & i & 26 & 24 \\
\hline 38 & | & états & | & 0.99999 & | & 57 & 40 \\
\hline 39 & i & rangs & i & 0.99999 & i & 45 & 34 \\
\hline 40 & | & nations & i & 0.99999 & i & 34 & 28 \\
\hline 41 & i & membres & I & 0.99999 & i & 104 & 59 \\
\hline 42 & | & plans & 1 & 0.99999 & i & 35 & 28 \\
\hline 43 & i & côtés & i & 0.99999 & i & 39 & 30 \\
\hline 44 & | & volumes & I & 0.99999 & I & 74 & 45 \\
\hline 45 & i & points & i & 0.99999 & i & 427 & 166 \\
\hline 46 & | & variétés & | & 0.99999 & 1 & 43 & 31 \\
\hline 47 & | & phases & | & 0.99999 & i & 24 & 21 \\
\hline 48 & i & sociétés & i & 0.99999 & i & 44 & 31 \\
\hline 49 & i & voix & i & 0.99999 & i & 111 & 58 \\
\hline 50 & i & villes & i & 0.99999 & i & 78 & 45 \\
\hline 51 & 1 & chambres & I & 0.99999 & i & 41 & 29 \\
\hline 52 & i & genres & i & 0.99999 & i & 22 & 19 \\
\hline 53 & 1 & voies & i & 0.99999 & i & 38 & 27 \\
\hline 54 & 1 & ministres & I & 0.99999 & i & 24 & 20 \\
\hline 55 & i & religions & i & 0.99999 & i & 14 & 14 \\
\hline 56 & 1 & doubles & । & 0.99999 & i & 14 & 14 \\
\hline 57 & i & corps & i & 0.99999 & i & 41 & 28 \\
\hline 58 & i & types & i & 0.99999 & i & 117 & 58 \\
\hline 59 & i & races & i & 0.99999 & i & 23 & 19 \\
\hline 60 & i & occasions & i & 0.99999 & 1 & 40 & 27 \\
\hline 61 & i & personnages & i & 0.99999 & i & 70 & 39 \\
\hline 62 & | & exemplaires & i & 0.99999 & i & 86 & 45 \\
\hline 63 & | & condamnations & 1 & 0.99999 & i & 12 & 12 \\
\hline 64 & i & compartiments & i & 0.99999 & i & 12 & 12 \\
\hline 65 & i & vue & i & 0.99999 & i & 19 & 16 \\
\hline 66 & 1 & ouvrages & i & 0.99999 & i & 81 & 42 \\
\hline 67 & 1 & épaisseurs & । & 0.99999 & । & 16 & 14 \\
\hline 68 & | & sciences & 1 & 0.99999 & i & 18 & 15 \\
\hline 69 & | & familles & i & 0.99999 & i & 107 & 50 \\
\hline 70 & | & amants & । & 0.99999 & । & 15 & 13 \\
\hline 71 & i & dizaines & i & 0.99999 & i & 292 & 109 \\
\hline 72 & i & significations & i & 0.99999 & i & 10 & 10 \\
\hline 73 & | & cycles & 1 & 0.99999 & i & 10 & 10 \\
\hline 74 & | & formes & 1 & 0.99999 & i & 83 & 41 \\
\hline 75 & i & communes & i & 0.99999 & i & 39 & 24 \\
\hline 76 & | & termes & i & 0.99999 & i & 47 & 27 \\
\hline 77 & | & époques & । & 0.99999 & | & 12 & 11 \\
\hline 78 & 1 & séjours & 1 & 0.99999 & i & 12 & 11 \\
\hline 79 & 1 & cylindres & 1 & 0.99999 & 1 & 12 & 11 \\
\hline 80 & 1 & domestiques & 1 & 0.99999 & । & 24 & 17 \\
\hline 81 & 1 & laboratoires & I & 0.99999 & | & 9 & 9 \\
\hline 82 & 1 & branches & । & 0.99999 & I & 88 & 41 \\
\hline 83 & 1 & organes & 1 & 0.99998 & । & 19 & 14 \\
\hline 84 & i & partis & i & 0.99998 & i & 13 & 11 \\
\hline 85 & | & existences & । & 0.99998 & 1 & 17 & 13 \\
\hline 86 & 1 & étapes & । & 0.99998 & i & 33 & 20 \\
\hline 87 & 1 & couleurs & I & 0.99998 & I & 33 & 20 \\
\hline 88 & । & chevaux & i & 0.99998 & i & 38 & 22 \\
\hline 89 & । & gènes & i & 0.99998 & i & 8 & 8 \\
\hline 90 & 1 & enfants & I & 0.99998 & I & 134 & 55 \\
\hline 91 & । & directions & | & 0.99997 & | & 29 & 18 \\
\hline
\end{tabular}




\begin{tabular}{|c|c|c|c|c|c|c|}
\hline 92 & I centres & I & 0.99997 & I & 29 & 18 \\
\hline 93 & périodes & | & 0.99997 & i & 20 & 14 \\
\hline 94 & domaines & 1 & 0.99997 & i & 27 & 17 \\
\hline 95 & versions & । & 0.99997 & 1 & 10 & 9 \\
\hline 96 & circonscriptions & | & 0.99997 & i & 10 & 9 \\
\hline 97 & bureaux & i & 0.99997 & i & 18 & 13 \\
\hline 98 & degrés & i & 0.99996 & i & 103 & 44 \\
\hline 99 & atomes & | & 0.99995 & 1 & 23 & 15 \\
\hline 100 & projets & 1 & 0.99994 & i & 28 & 17 \\
\hline 101 & fonctions & 1 & 0.99994 & i & 21 & 14 \\
\hline 102 & | métaux & | & 0.99993 & i & 7 & 7 \\
\hline 103 & crans & i & 0.99993 & i & 7 & 7 \\
\hline 104 & tonnes & i & 0.99993 & i & 26 & 16 \\
\hline 105 & | députés & | & 0.99992 & i & 31 & 18 \\
\hline 106 & | noyaux & | & 0.99989 & i & 15 & 11 \\
\hline 107 & I maladies & i & 0.99989 & i & 15 & 11 \\
\hline 108 & lots & 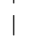 & 0.99989 & i & 9 & 8 \\
\hline 109 & listes & 1 & 0.99989 & i & 9 & 8 \\
\hline 110 & I dialectes & i & 0.99989 & i & 9 & 8 \\
\hline 111 & | comités & i & 0.99989 & i & 9 & 8 \\
\hline 112 & | chemins & i & 0.99988 & i & 13 & 10 \\
\hline 113 & | salles & 1 & 0.99988 & i & 22 & 14 \\
\hline 114 & rangées & 1 & 0.99988 & i & 22 & 14 \\
\hline 115 & I machines & i & 0.99988 & i & 22 & 14 \\
\hline 116 & voyages & i & 0.99983 & 1 & 30 & 17 \\
\hline 117 & | témoins & i & 0.99983 & i & 25 & 15 \\
\hline 118 & cordes & 1 & 0.99980 & i & 18 & 12 \\
\hline 119 & I journaux & 1 & 0.99977 & i & 98 & 40 \\
\hline 120 & | sessions & | & 0.99975 & | & 6 & 6 \\
\hline 121 & | quantités & 1 & 0.99975 & | & 6 & 6 \\
\hline 122 & | orifices & i & 0.99975 & i & 6 & 6 \\
\hline 123 & | mécanismes & i & 0.99975 & i & 6 & 6 \\
\hline 124 & issues & | & 0.99975 & i & 6 & 6 \\
\hline 125 & | armées & । & 0.99975 & i & 6 & 6 \\
\hline 126 & bandes & i & 0.99969 & i & 26 & 15 \\
\hline 127 & | caractères & i & 0.99967 & i & 48 & 23 \\
\hline 128 & I rapports & i & 0.99966 & i & 51 & 24 \\
\hline 129 & | organisations & । & 0.99963 & i & 8 & 7 \\
\hline 130 & | hivers & । & 0.99963 & i & 8 & 7 \\
\hline 131 & guerres & | & 0.99963 & 1 & 8 & 7 \\
\hline 132 & fins & | & 0.99963 & i & 8 & 7 \\
\hline 133 & | hypothèses & । & 0.99962 & i & 29 & 16 \\
\hline 134 & I de & । & 0.99962 & i & 29 & 16 \\
\hline 135 & I routes & । & 0.99961 & i & 10 & 8 \\
\hline 136 & | quartiers & | & 0.99959 & 1 & 24 & 14 \\
\hline 137 & | régions & | & 0.99955 & 1 & 46 & 22 \\
\hline 138 & I auteurs & i & 0.99952 & i & 124 & 47 \\
\hline 139 & substances & i & 0.99944 & i & 22 & 13 \\
\hline 140 & circonstances & i & 0.99936 & i & 65 & 28 \\
\hline 141 & | compagnies & i & 0.99932 & | & 33 & 17 \\
\hline 142 & sources & | & 0.99932 & | & 15 & 10 \\
\hline 143 & | propriétaires & | & 0.99932 & | & 15 & 10 \\
\hline 144 & ordres & | & 0.99927 & | & 81 & 33 \\
\hline 145 & entreprises & | & 0.99926 & 1 & 36 & 18 \\
\hline 146 & portes & | & 0.99918 & 1 & 28 & 15 \\
\hline 147 & | paires & | & 0.99918 & i & 28 & 15 \\
\hline 148 & tours & | & 0.99914 & | & 144 & 52 \\
\hline 149 & I étés & | & 0.99914 & 1 & 13 & 9 \\
\hline 150 & I attaques & | & 0.99914 & | & 13 & 9 \\
\hline
\end{tabular}

Tableau 3 : N spécifiques de plusieurs

Certains chiffres de cette seconde liste peuvent surprendre. Prenons le cas de la ligne numéro $7:$ on constate que sur 714 occurrences, seules 305 correspondent au syntagmes plusieurs personnes, c'est-à- 
dire moins de la moitié. On peut alors s'étonner du taux de spécificité associé à personnes puisqu'il y a plus d'occurrences du syntagme quelques personnes dans le corpus C. L'explication de ce classement est relativement simple. Il suffit de se rappeler que les sous-corpus Q (63098 phrases) et P (21009 phrases) ne sont pas de taille égale mais qu'au contraire, Q ([quelques $N]$ ) est trois fois plus important que $\mathrm{P}$ ([plusieurs $N]$ ). Par conséquent, si plusieurs personnes compte un peu moins de la moitié des occurrences totales de personnes, cela représente toutefois une proportion très importante si l'on tient compte de la différence de taille entre $\mathrm{Q}$ et $\mathrm{P}$.

Avant d'entrer dans l'analyse des données, précisons que de telles mesures constituent un élément d'enquête utile, mais qu'elles doivent être employées avec prudence. Comme nous allons le montrer, tous les substantifs spécifiques d'un déterminant ne le sont pas pour les mêmes raisons et les chiffres obtenus ne doivent pas être pris au pied de la lettre. Par exemple, parmi les spécificités de plusieurs (Tableau 3), la présence de étages (rang numéro 1) n'a pas la même explication que celle de sortes (rang numéro 4). En outre, si vers est une forme spécifique de quelques, ce n'est pas pour autant que le syntagme plusieurs vers est impossible. On ne peut nier l'existence d'au moins deux occurrences de plusieurs vers dans $\mathrm{C}$, et au-delà, le caractère parfaitement naturel de ce syntagme ${ }^{7}$. Néanmoins, la surreprésentation de vers après quelques et sa sous-représentation après plusieurs montrent qu'il y a là une contrainte qui s'applique et qui doit être explicitée. C'est l'objectif de la section suivante.

\section{Quelques et plusieurs : un mode de sommation différent}

\subsection{Présentation des données}

Une première série d'exemples, que nous avons identifiée à partir des spécificités positives de quelques (et négatives de plusieurs), permet de différencier les deux déterminants de façon très nette ${ }^{8}$ :

7. a. Nous avons fait quelques pas. (Tableau 2, rang 5)

b. Il est tombé quelques \{gouttes / flocons\}. (rang 16)

c. J'ai dit quelques \{mots / phrases\} / On a échangé quelques \{mots / phrases\}. (rangs 9 et 4)

d. Nous avons échangé \{quelques / ?plusieurs\} paroles. (rang 6)

8. a. Nous avons fait plusieurs pas. (*une marche)

b. Il est tombé plusieurs \{gouttes / flocons\}. (*une averse)

c. J'ai dit plusieurs \{mots / phrases\} (*un discours) / On a échangé plusieurs \{mots / ?phrases\}. (échanger des messages / des insultes)

d. Pour le mariage, plusieurs paroles du Christ, indiquent sa volonté d'élever l'institution naturelle du mariage à l'état de sacrement.

Dans 7a, le prédicat verbal faire quelques pas peut être synonyme de faire une petite marche, une petite promenade, alors que cette interprétation est impossible avec faire plusieurs pas. Le même principe s'applique dans $7 \mathrm{~b}$ où il tombe quelques gouttes / flocons peut signifier pleuvoir / neiger, alors que il tombe plusieurs gouttes / flocons ne peut pas prendre ce sens. Or, le rapport entre un certain nombre de pas et un nom d'activité tel que marche est identique à celui qui existe entre un certain nombre de gouttes et une averse. Dans les deux cas, le procès global (marcher ou pleuvoir) correspond à la sommation (indéfinie) d'éléments identiques (des pas ou des gouttes).

On retrouve aussi ce rapport dans l'exemple 7c entre un procès tel que discourir ou discuter et une sommation (indéfinie) de mots ou de phrases. A nouveau, le SN déterminé par quelques peut renvoyer au procès global (discourir, discuter) alors que c'est impossible avec plusieurs. Il est intéressant de noter que cette impossibilité se traduit, en 8c, par un changement du sens de mots, qui devient synonyme de messages ou d'insultes dans ce contexte.

On retrouve un fonctionnement analogue dans l'exemple $7 \mathrm{~d}$, où quelques paroles peut correspondre à une courte discussion, tandis que plusieurs paroles n'est pas naturel dans le contexte $8 \mathrm{~d}$. Ce dernier exemple est intéressant dans la mesure où il constitue un cas limite en termes d'attestabilité : on ne trouve que 2 
occurrences du syntagme plusieurs paroles sur l'ensemble de Frantext catégorisé (contre 469 occurrences de quelques paroles) et ce déséquilibre hisse paroles tout en haut des spécificités positives de quelques. Comme nous l'avons déjà souligné (et comme nous aurons l'occasion d'y revenir), cela n'implique pas que l'expression plusieurs paroles soit impossible, bien au contraire. Mais les occurrences - beaucoup moins nombreuses - de plusieurs paroles ne font jamais référence à une courte discussion; elles présentent un sens particulier de paroles qui peut renvoyer aux écrits religieux (exemple 8d), aux textes de chansons («ce site propose plusieurs paroles (de chansons)»), ou encore à des promesses verbales («il a plusieurs paroles »).

Sur la base de ces premiers exemples, nous allons avancer un principe informel permettant de différencier les deux déterminants et dont la suite de ce travail nous permettra d'interroger et de vérifier la validité : quelques $N$ désignerait un ensemble d'éléments pris dans sa globalité, ou pour le dire autrement, un collectif. Plusieurs $N$, au contraire, désignerait une sommation d'éléments distincts sans qu'il soit question de les intégrer dans un cadre plus global ${ }^{9}$. Un nouvel exemple permet d'illustrer ce principe :

9. a. A l'enterrement, il a versé quelques larmes. (rang 18)

b. ?A l'enterrement, il a versé plusieurs larmes.

Dans 9a quelques larmes dénote une sommation de larmes prises dans leur ensemble, de telle sorte que leur accumulation finit par renvoyer à un procès global, à savoir pleurer. Cela est clairement impossible avec plusieurs, comme on le voit dans $9 \mathrm{~b}$, puisque les larmes sont prises individuellement et rendent la phrase pour le moins étrange : on a le sentiment que les larmes sont versées au compte-goutte.

D'une manière générale, il suffit que la sommation des atomes soit conçue comme un tout pour que l'utilisation de plusieurs devienne problématique. Ainsi, dans l'exemple 10b, le fait que chaque rire ou chaque pleur soit présenté individuellement et distinctement des autres paraît bizarre, alors qu'avec quelques, nous n'avons pas ce problème :

10. a. \{Quelques / ?Plusieurs\} rires ont éclaté parmi les étudiants. (rang 123)

b. Après \{quelques / ?plusieurs\} pleurs, le jeune homme se consola bien vite en buvant une bouteille de rhum. (rang 136)

Notre hypothèse de travail peut, en outre, s'étendre au-delà des procès, comme on le constate dans les exemples suivants :

11. a. Il lui reste quelques cheveux. (rang 46)

b. Il a quelques poils (de barbe) sur les joues. (rang 60)

c. Il travaille pour quelques euros / sous. (rang 2)

d. Il a quelques connaissances en médecine. (rang 114)

e. Il a quelques notions en mathématiques. (rang 45)

12. a. ?Il lui reste plusieurs cheveux.

b. ?Il a plusieurs poils (de barbe) sur les joues.

c. ?Il travaille pour plusieurs euros / sous.

d. Il a plusieurs connaissances en médecine.

e. ?Il a plusieurs notions en mathématiques.

f. On rencontre ici plusieurs notions capitales.

Pour les exemples 11a et 12a, Gondret (1976, p. 147) propose l'explication suivante: «on emploie quelques (et non plusieurs) avec ce qui se présente habituellement en collections assez nombreuses ». Or, nous avons montré que cette approche, qui tente d'ordonner quelques et plusieurs sur l'échelle de la quantité, donne des résultats contradictoires et pose plus de problèmes qu'elle n'en résout. Le principe de différenciation que nous avançons, en revanche, rend directement compte de ces exemples. Dans 11a, quelques cheveux désigne un ensemble de cheveux pris globalement. Cette prise en compte globale se traduit par un changement d'échelle qui nous fait passer d'une simple sommation de cheveux à une collectivité à part entière, c'est-à-dire une chevelure, même si celle-ci est peu fournie ${ }^{10}$. En substituant plusieurs à quelques dans ce contexte, au contraire, nous renvoyons à une sommation de cheveux 
individuels, sans que cette sommation puisse prétendre au statut de collectif. Le raisonnement est le même pour $11 \mathrm{~b}$ où seul quelques poils peut renvoyer à une (petite) barbe, contrairement à plusieurs poils qui n'est pas naturel dans ce contexte.

Ce changement de perspective semble être aussi à l'œuvre dans l'exemple 11c, puisque quelques euros peut désigner une somme d'argent, contrairement à plusieurs euros qui est plus étonnant ${ }^{11}$.

Notre hypothèse de travail peut aussi s'appliquer à des noms abstraits, comme on le voit dans l'exemple 11. Quelques connaissances est ambigu et peut aussi bien désigner un savoir (restreint) qu'un groupe de personnes connues du locuteur. Au contraire, si le syntagme plusieurs connaissances désigne un groupe de relations, il ne peut renvoyer à un savoir. Le raisonnement est identique pour quelques / plusieurs notions (exemples 11e et 12e).

A partir de ces exemples, nous pouvons faire un certain nombre de remarques et préciser notre hypothèse de travail. Tout d'abord, il faut relativiser l'importance de la quantité désignée par les deux déterminants. La question que l'on se pose habituellement et qui consiste à se demander si plusieurs renvoie à une quantité un peu plus grande que quelques ou si c'est le contraire est en effet peu pertinente : y a-t-il un sens à se demander si le nombre de gouttes dont on a besoin pour renvoyer à une petite pluie est supérieur ou non au nombre de gouttes désigné par le quantificateur plusieurs gouttes? Au contraire, ces exemples montrent que la différence entre quelques et plusieurs tient moins à une question de quantité qu'à la manière dont est présentée la sommation des atomes. La commutation des deux déterminants implique un changement de perspective qui donne accès, dans le cas de quelques, à ce qu'on pourrait appeler, dans le cadre de la sémantique formelle, un groupe, c'est-à-dire une entité collective qui ne se réduit pas à la simple sommation de ses atomes, alors que dans le cas de plusieurs, nous avons simplement affaire à un ensemble (ou une somme d'éléments individuels) ${ }^{12}$.

\subsection{Quelques $\mathbf{N}$ : créateur de collectif réduit}

D'autres faits vont dans le sens de notre analyse. Lorsqu'on observe les spécificités de quelques, on est frappé par la présence d'une classe de $\mathrm{N}$ qui ont en commun la possibilité de désigner les éléments d'une collection (désormais, Nélém). L'exemple de vers (Tableau 2, rang 1), en tant qu'élément d'une poésie en est une bonne illustration. Mais d'autres exemples, tirés du Tableau 2, illustrent aussi ce point, comme notes (dont les occurrences correspondent à des notes de musiques), marches (parties d'un escalier), traits (traits d'un dessin ou d'un caractère), mais aussi lignes, fleurs, arbres, etc. Le tableau suivant donne pour chaque Nélém identifié (colonne lemme) sa spécificité (calculée, rappelons-le, en fonction de la taille respective des corpus $\mathrm{Q}$ et $\mathrm{P}$ ), le nombre d'occurrences après quelques (colonne subfreq) et le nombre d'occurrences total (freq). 


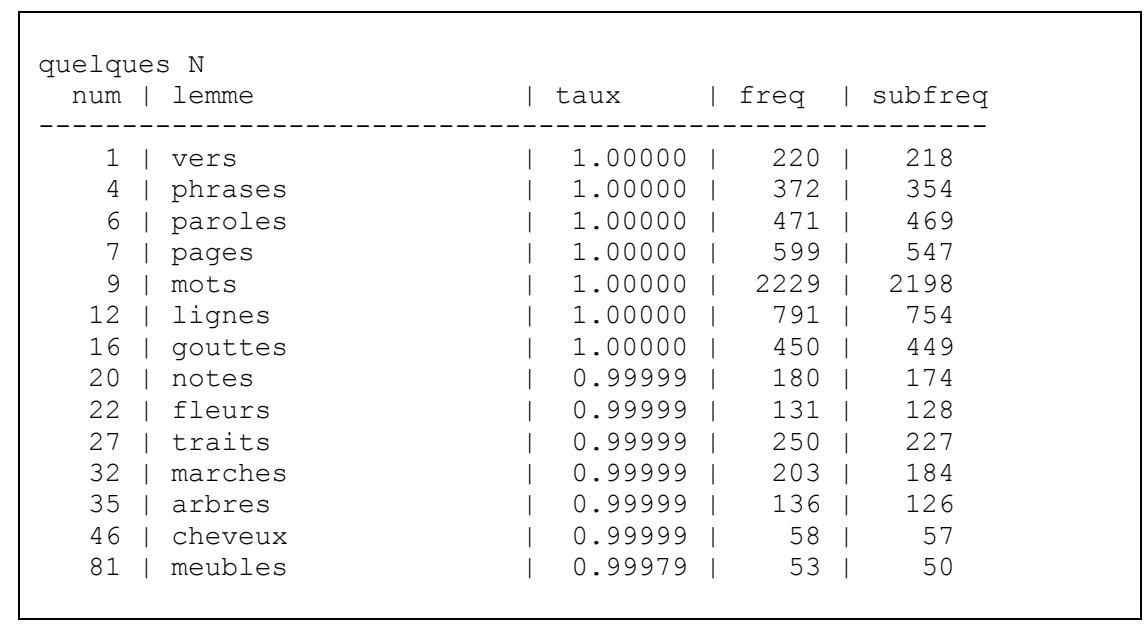

Tableau 4 : quelques et les $\mathrm{N}$ d'éléments

Ainsi, de la même manière que le syntagme quelques cheveux peut être amené, dans certaines conditions, à désigner une petite chevelure, quelques vers / pages / lignes / notes / traits / marches, etc. peuvent désigner respectivement un(e) petit(e) poème / fascicule / message / air de musique /dessin / escalier.

Comme de nombreux auteurs l'ont souligné dans le cadre des recherches portant sur les noms collectifs et sur la relation membre / collection, un type d'éléments peut correspondre à différentes collections. Une pluralité de vers peut ainsi renvoyer à un quatrain, un bout-rimé, une poésie ; une pluralité d'arbres peut renvoyer à une forêt, un bosquet, une futaie, un bois ; une pluralité de pages peut renvoyer à un fascicule, un carnet, un livret. Il n'est donc pas possible de déterminer le collectif qui commuterait exactement avec l'expression collective quelques $N$, mais il n'en demeure pas moins que dans certains cas, l'entité désignée par le syntagme quelques $N$ a des propriétés qui le rapprochent d'un nom collectif en se contentant d'y ajouter une restriction (sur le nombre d'éléments ou la taille du collectif).

Les exemples suivants confirment cela en montrant qu'il est tout à fait possible de prédiquer sur la collectivité dénotée par quelques $N$ et non sur chaque élément pris individuellement, alors que plusieurs, au contraire, impose une lecture distributive dans des contextes identiques :

\section{3. a. J'ai envoyé \{quelques /*plusieurs\} lignes à ma famille ( $\rightarrow$ J'ai envoyé un petit message) \\ b. Il nous a interprété \{quelques /*plusieurs\} notes de Bach ( $\rightarrow$ Il nous a interprété un petit air) \\ c. Laissez-moi vous offrir \{quelques /*plusieurs\} fleurs ( $\rightarrow$ un petit bouquet) \\ d. \{Quelques /*Plusieurs\} gouttes affecteront la pointe du Finistère ( $\rightarrow$ une petite pluie)}

Ainsi, deux expressions comme écrire quelques lignes et écrire plusieurs lignes sont parfaitement naturelles, mais seule la première a cette possibilité supplémentaire de désigner un (court) message, c'està-dire un tout constitué d'une sommation indéfinie de lignes écrites. Dès lors, le prédicat envoyer, qui s'applique au message, et non aux lignes dont il est constitué, ne pose aucun problème dans le contexte de 13a. A l'inverse, plusieurs lignes présente ces lignes de manière individuelle, sans qu'il soit fait état d'une entité collective dont ces lignes seraient les atomes. Le prédicat envoyer n'est donc plus adapté, car si l'on peut envoyer un message, ce n'est pas pour autant qu'on envoie des lignes.

Dans le même ordre d'idées, si le verbe interpréter ne peut pas s'appliquer aux notes qui composent un air de musique, il peut en revanche porter sur quelques notes (exemple 13b). C'est bien la preuve que quelques notes désigne un petit air de musique en tant que tel et non une simple pluralité de notes. Les deux derniers exemples fonctionnent selon le même principe. En offrant quelques fleurs, on n'offre pas chaque fleur individuellement mais bien un petit bouquet, et on conçoit aisément que seule une pluie authentique, et non une pluralité de gouttes, est capable d'affecter la pointe du Finistère. 
Si ce point permet d'opposer quelques à plusieurs, notons au passage qu'il permet aussi de distinguer quelques et $\operatorname{des}^{13}$ :

\title{
14. a. *J'ai envoyé des lignes à ma famille. \\ b. *Il nous a interprété des notes de Bach. \\ c. Laissez-moi vous offrir des fleurs. \\ d. *Des gouttes affecteront la pointe du Finistère.
}

Le fait que [quelques Nélém.] puisse directement désigner un collectif constitué de Nélém. nous permet d'expliquer les taux de spécificité observés dans le Tableau 4. En effet, puisque quelques arbres a cette possibilité supplémentaire de renvoyer à un (petit) bois / bosquet, contrairement à plusieurs arbres, alors les occurrences de arbres à droite de quelques dans le corpus total augmentent d'autant. Du coup, la forme arbres en vient à être surreprésentée dans le sous-corpus $\mathrm{Q}$ et finit par obtenir un taux de spécificité considérable. Comme pour beaucoup des noms qui figurent dans les listes de spécificités, le fait que arbres soit spécifique de quelques ne s'explique pas parce qu'il ne peut pas apparaître après plusieurs (il est au contraire parfaitement naturel dans ce contexte), mais parce qu'associé à quelques, il a une possibilité d'emploi supplémentaire qu'il n'a pas avec plusieurs.

Le mécanisme illustré par les exemples 13 n'est évidemment possible qu'à condition que le collectif [quelques Nélém.] soit exclusivement constitué d'éléments dénotés par Nélém. Ainsi, quelques villageois ne peut pas désigner un « petit village » pour la bonne et simple raison qu'un village n'est pas seulement constitué de villageois, mais aussi de maisons, de rues, etc.; de même, quelques wagons ne peut pas désigner un «petit train » puisqu'un train est aussi constitué d'une locomotive; enfin, quelques perles ne désignera pas un «petit collier» puisqu'il faut aussi un fil. De ce point de vue, le collectif dénoté par quelques $N$ respecte l'une des propriétés fondamentales des noms collectifs, à savoir l'homogénéité interne $^{14}$. L'une des conséquences de cela, c'est que quelques $N$ ne peut pas renvoyer à cette classe particulière de N que Flaux (1999) et Flaux \& Van de Velde (2000) appellent des noms de totalité : train, livre, chapelet, collier, etc.

Pour finir, précisons que les autres relations méronymiques impliquant une homogénéité interne ne peuvent pas non plus être reconstruites grâce au syntagme quelques $N$. Ainsi, quelques tranches ne feront jamais une "petite brioche » ou un "petit gâteau ». C'est que la relation mise en œuvre ici est une relation portion / masse, et non une relation membre / collection ${ }^{15}$.

\section{Quelques, plusieurs et les entités homogènes}

\subsection{Les $\mathbf{N}$ intrinsèquement pluriels (pluralia tantum)}

Sur la base des calculs de spécificités décrits supra et des exemples qui précèdent, nous avons établi l'existence d'un mode de sommation différent pour les indéfinis quelques et plusieurs. Dans les sections qui suivent, nous allons en observer les conséquences sur de nouvelles classes d'exemples. La première correspond à ce que la tradition grammaticale appelle les pluralia tantum.

Bosveld-de Smet (2000), qui parle de « $\mathrm{N}$ intrinsèquement pluriels », montre à leur propos qu'ils doivent être rapprochés des massifs :

\begin{abstract}
«La plupart de ces $\mathrm{N}$ se rangent du côté des $\mathrm{N}$ de masse plutôt que de celui des $\mathrm{N}$ comptables. L'entité désignée n'est souvent pas perçue comme une entité divisible en individus distincts, discernables les uns des autres. Or, ce fait se reflète par les contraintes que ces $\mathrm{N}$ imposent aux déterminants qui peuvent les précéder. On mange des mais non pas plusieurs épinards, on jette les mais non pas trois entrailles, et on donne à quelqu'un des et non pas différents étrennes. » Bosveld-de Smet (2000, p. 373)
\end{abstract}

Le critère principal permettant d'identifier ces unités tient donc dans le fait qu'elles ne peuvent être précédées d'un numéral et qu'elles ne peuvent pas apparaître au singulier sans changer de sens. Or, ce que 
Bosveld-de Smet ne mentionne pas, c'est que seul quelques, et non plusieurs, a la possibilité de précéder de tels noms. Ce point est souligné dans Bacha (1997, p. 55) puis dans Leeman (2004, p. 169) qui parle, quant à elle, de «massifs pluriels » pour désigner ces noms :

15. a. Vous reprendrez bien \{des / quelques / *plusieurs/*huit\} \{haricots / épinards / pâtes / rillettes\}.

b. J'ai \{des / quelques /*plusieurs/*huit\} \{économies / provisions\}.

c. Il faut vous attendre à \{des / quelques / *plusieurs/*huit\} représailles.

d. J'ai amené \{des / quelques /*plusieurs/*huit\} effets personnels.

e. Sa mère lui a laissé \{des / quelques / *plusieurs/*huit\} hardes.

f. $\{$ Des / Quelques / *Plusieurs/*Huit $\}$ décombres recouvrent le trottoir.

g. $\{$ Des / Quelques / *Plusieurs/*Huit\} flots viennent lécher le bord de la falaise.

Afin de confirmer l'existence de cette contrainte distributionnelle, nous avons fait l'inventaire des $\mathrm{N}$ intrinsèquement pluriels dans nos propres listes de spécificités et nous les avons regroupés dans le tableau suivant. Ce tableau permet de constater, par exemple, que sur 64 occurrences de débris, 62 apparaissent après quelques et 2 après plusieurs (première ligne du tableau, rang 42).

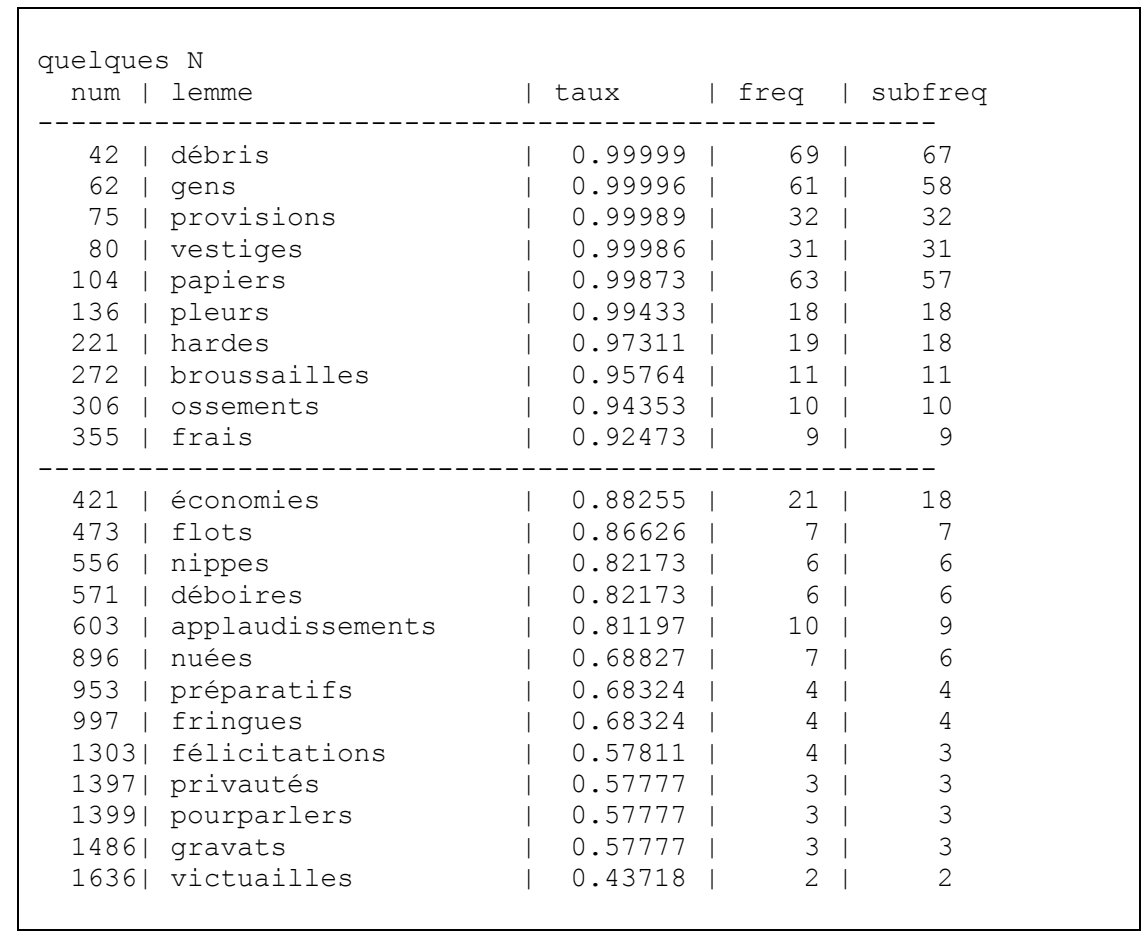

Tableau 5 : quelques et les $\mathrm{N}$ intrinsèquement pluriels

Précisons que le taux de spécificité n'est significatif qu'au dessus de 0.90. En dessous de ce seuil, on ne peut techniquement plus dire qu'une forme est spécifique d'un déterminant donné. C'est pourquoi nous séparons dans le tableau précédent les cas significatifs (de débris jusqu'à frais) des autres (à partir de économies) : ces derniers ont un nombre d'occurrences dans le corpus total trop faible pour qu'on puisse leur associer un taux de spécificité significatif. Toutefois, le fait de les mentionner permet d'observer que ces derniers fonctionnent de manière analogue aux premiers. Ils confirment donc la surreprésentation des pluralia tantum dans le contexte droit de quelques (et de leur sous-représentation dans le contexte de plusieurs). 
Une analyse détaillée des rares occurrences de pluralia tantum déterminées par plusieurs montre qu'ils doivent être, dans ce cas précis, rangés du côté des dénombrables. Ainsi, les deux occurrences de plusieurs débris concernent des débris humains et les occurrences de plusieurs gens présentent toutes un complément de nom : plusieurs gens du bourg, gens d'église ou gens du beau monde, alors qu'avec quelques la structure binominale n'est pas nécessaire : quelques gens s'empressent autour d'elle. Si les 18 occurrences de quelques hardes désignent quant à elles «l'ensemble des effets personnels que l'on emmène dans ses bagages » (TLFi) et mettent ainsi en jeu l'indénombrable hardes, l'unique occurrence de plusieurs hardes dans Frantext catégorisé sert à désigner des " troupes d'oiseaux ». Hardes est alors dénombrable (et varie en nombre). De même, plusieurs précède économies lorsqu'il s'agit de plusieurs économies nationales ou encore de plusieurs économies dominantes. Pour cet ensemble réduit d'exemples, le $\mathrm{N}$ perd donc automatiquement son statut de massif pour devenir comptable : trois débris humains, deux gens d'église, deux économies parallèles, quatre hardes de pécaris.

Le reste des occurrences va dans le sens de notre analyse. Les $\mathrm{N}$ intrinsèquement pluriels ne désignent pas une simple pluralité d'éléments, mais au contraire, présentent une structuration interne (plus ou moins homogène et discontinue) qui les rapproche beaucoup plus d'indénombrables comme riz ou gazon. Dans la mesure où les atomes sont difficilement discernables les uns des autres, il est tout à fait logique que quelques soit le seul à pouvoir les déterminer, puisque comme nous l'avons vu, il a cette possibilité supplémentaire de construire une entité collective qui ne se réduit pas à une simple sommation d'atomes. A l'opposé, plusieurs exige une sommation d'éléments bien individués pour pouvoir fonctionner correctement et tel n'est pas le cas des $\mathrm{N}$ intrinsèquement pluriels.

D'autres exemples, qui n'apparaissent pas dans Frantext, mais qui n'en sont pas moins fréquents dans d'autres discours (par exemple, journalistique), permettent d'approfondir le mécanisme :

16. a. Cette actrice a \{des / quelques\} \{rondeurs / formes\}.

b. ${ }^{*}$ Cette actrice a plusieurs \{rondeurs / formes\}.

Dans l'exemple 16a, le syntagme quelques rondeurs ne désigne pas une simple pluralité de rondeurs, mais une caractéristique globale de l'actrice (à savoir, une certaine corpulence). En cela, il s'oppose clairement au quantificateur plusieurs rondeurs qui désigne bien une sommation d'éléments distincts (où chaque rondeur est prise séparément) et qui du coup, s'interprète plus difficilement dans ce contexte. Le raisonnement est identique lorsqu'on compare avoir quelques formes, qui dénote à nouveau une propriété globale, à l'expression avoir plusieurs formes qui désigne une pluralité de formes distinctes (carrée, circulaire, conique, cylindrique, etc.), ce qui n'est pas non plus très naturel dans le contexte $16 \mathrm{~b}$.

Pour finir, nous pouvons évoquer le cas d'une catégorie de N qui, au pluriel, « ont un sens différent de leur singulier correspondant. » Bosveld-de Smet (2007, p. 365). Il s'agit de $\mathrm{N}$ tels que papiers, épinards, ou pâtes que les grammaires ne mentionnent généralement pas comme pluralia tantum, bien qu'ils présentent une structuration interne comparable. L'exemple 17 montre qu'ils ne font pas exception, puisque quelques pâtes peut désigner un collectif (un plat) composé d'une sommation indéfinie d'atomes (chaque pâte), alors que dans un contexte identique, plusieurs pâtes (cf. exemple 17b) est plutôt amené à désigner des sortes de pâtes, à savoir des Cannellonis, des Macaronis, des Farfalles, des Pennes, des Spaghettis, des Tagliatelles, des Torsades, des Orecchiettes, des Cappelli di angelo, des Conchiglie et des Lasagnes $^{16}$ :

17. a. Ce soir, on mange quelques pâtes. (plat)

b. Ce soir, on mange plusieurs pâtes. (types de pâtes)

\subsection{Les entités homogènes continues}

Une dernière série de noms surreprésentés après quelques retiendra notre attention dans ce travail, à savoir les noms de temporalité moment et instant ${ }^{17}$, dont on peut trouver une caractérisation sémantique dans Kleiber (1997). Ces $\mathrm{N}$ doivent être classés parmi les dénombrables, puisqu'ils sont sensibles à l'opposition singulier / pluriel et qu'ils n'acceptent pas le partitif. Ils ont toutefois des propriétés communes avec les indénombrables qui font d'eux des comptables atypiques. Ainsi, le test de divisibilité 
homogène, que l'on applique classiquement aux massifs (si on enlève de l'eau à de l'eau, c'est toujours de l'eau), s'applique exactement dans les mêmes termes pour moment et instant (si on enlève un instant à un instant, c'est encore un instant). Par ailleurs, l'opération inverse, à savoir le test de référence cumulative (si on ajoute de l'eau à de l'eau, c'est encore de l'eau), est lui aussi applicable : «toute addition de parties qui sont des moments sera elle-même un moment» Kleiber (1997, p. 323). Or, c'est justement dans cette acception homogène que de tels $\mathrm{N}$ peuvent apparaitre après quelques :

18. Les deux amis restent \{quelques / *plusieurs\} \{moments / instants\} l'un à côté de l'autre.

Dans cet exemple, quelques instants dénote non pas une pluralité d'instants, mais bien un instant - peut être un peu plus long - mais un instant tout de même. Cela s'explique par le fait qu'une pluralité d'instants revient, sous la portée de quelques, à fusionner, fusion qui n'est rendue possible que par la structuration interne homogène de instant. Plusieurs, au contraire, impose une lecture atomisante de telle sorte que chaque moment / instant est clairement distingué des autres (c'est le cas de 19a), ou encore, de telle façon qu'il devient synonyme de étape (exemple 19b), bloquant définitivement toute forme de référence cumulative (une étape plus une étape, ça n'est plus une étape).

19. a. J'ai passé \{quelques / plusieurs\} \{moments / instants\} sympathiques avec lui.

b. C'est l'ouverture infinie du vécu, signifiée à \{?quelques / plusieurs\} \{moments / instants\} de l'analyse husserlienne par la référence à une Idée au sens kantien.

Or il est intéressant de constater que ce principe s'observe aussi, quoique dans une moindre mesure, sur les dénombrables homogènes ayant une extension spatiale, comme nuage ou geste :

20. a. Demain, il y aura quelques nuages sur la France. (rang 40)

b. Ce matin, Pierre est sorti du coma. Il a fait quelques gestes. (rang 55)

Dans l'exemple 20a le fait que quelques nuages puisse s'étendre à la dimension d'un pays montre qu'il ne s'agit pas d'une simple addition de nuages mais d'une propriété globale que l'on pourrait gloser par « un ciel légèrement nuageux ». Dans ce cas, l'homogénéité interne des nuages rend possible une référence cumulative grâce à laquelle un nuage plus un nuage donne un (plus gros) nuage. Dans 20b, on observe le même principe : quelques gestes revient, par fusion des gestes, à un seul geste.

Avec plusieurs, au contraire, la référence cumulative semble beaucoup plus difficile :

21.a. ?Demain, il y aura plusieurs nuages sur la France.

b. Ce matin, Pierre est sorti du coma. Il a fait plusieurs gestes.

L'exemple 21a, en effet, parait moins naturel, et dans $21 \mathrm{~b}$, la meilleure interprétation reste celle où les gestes, au lieu de fusionner en un seul geste, s'opposent entre eux et correspondent ainsi à une véritable tentative de communication de la part de Pierre.

\section{Ouvertures}

D'autres éléments, que nous ne développerons pas dans ce travail, permettent de renforcer le point de vue défendu ici. Par exemple, Dobrovie-Sorin \& Beyssade (2005, p. 116) montrent que quelques et plusieurs s'opposent respectivement par une lecture collective et distributive, ce qui expliquerait, selon elles, les distributions suivantes :

\section{2. a. Plusieurs enfants étaient intelligents / tristes / blonds / fatigués. \\ b. ??Quelques enfants étaient intelligents. \\ c. ?Quelques enfants étaient tristes / blonds / fatigués. \\ d. Plusieurs / quelques enfants se ressemblaient.}

En outre, une structure syntaxique particulière est à l'origine d'une distribution complémentaire sur quelques et plusieurs. Il s'agit du complément circonstanciel de forme [en quelques / plusieurs $N$ ] qui sélectionne des substantifs très différents selon le déterminant. 
23. a. Il a terminé sa soupe en \{quelques / *plusieurs\} minutes.

b. Il a résumé la situation en \{quelques / *plusieurs\} \{mots / phrases\}.

c. En \{quelques / *plusieurs\} bonds, il parvint à la fenêtre.

On sait que cette structure sert classiquement de critère pour identifier un procès perfectif ${ }^{18}$. Dans le cas qui nous intéresse, en quelques $N$ caractérise ainsi le temps pris pour qu'un processus s'achève. Or, cela n'est pas possible avec plusieurs ${ }^{19}$. Une explication consisterait à dire que quelques $N$, en dénotant une collectivité de $\mathrm{N}$ plutôt qu'une pluralité, présenterait une clôture que n'a pas plusieurs $N$, clôture qui permettrait alors de marquer l'aspect télique du procès.

Par ailleurs, il faut souligner que le présent travail s'appuie principalement sur une analyse des spécificités positives de quelques (et donc, des spécificités négatives de plusieurs). Or, les spécificités positives de plusieurs (Tableau 3) présentent elles aussi des caractéristiques remarquables que nous n'avons pas la place d'exploiter en détail ici. Nous nous contenterons d'en mentionner quelques-unes dans ce qui suit.

Par exemple, si plusieurs sortes ne pose aucun problème, quelques sortes est impossible en français, et cette impossibilité se trouve confirmée dans le Tableau 3 au rang 4, où les 68 occurrences de sortes apparaissent toutes devant plusieurs. On pourrait être tenté d'expliquer cette asymétrie par la concurrence entre la forme plurielle (*quelques sortes) et la locution en quelque sorte (normalement au singulier). Or, loin de constituer un problème local, les listes de spécificités montrent que les $\mathrm{N}$ taxinomiques sont surreprésentés après plusieurs :

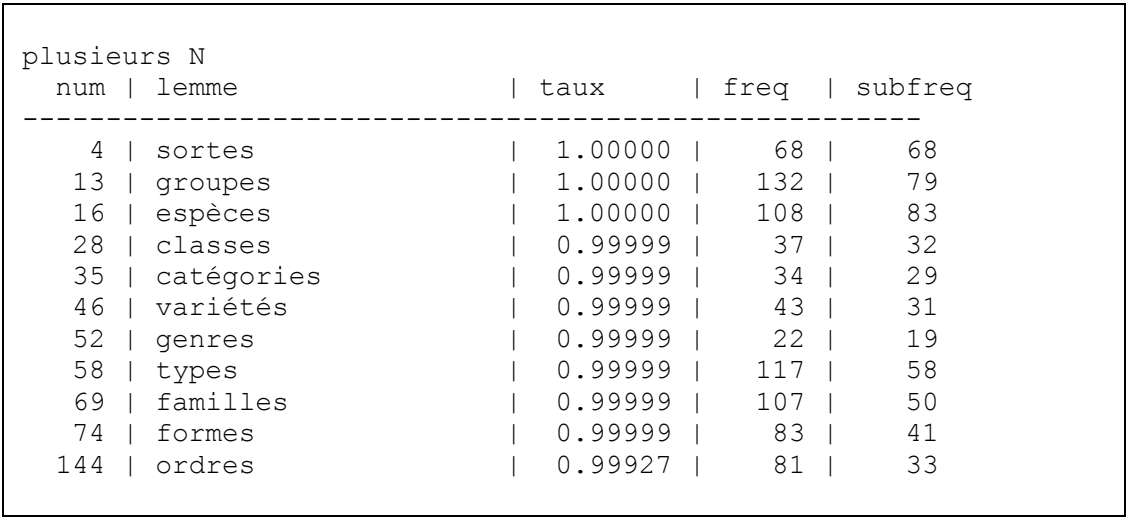

Tableau $6:$ quelques et les $\mathrm{N}$ intrinsèquement pluriels

Il est en effet facile de constater que certains contextes tendent clairement à privilégier le déterminant plusieurs au détriment de quelques ${ }^{20}$ :

24. a. J'utilise \{plusieurs / *quelques\} moyens de locomotion pour me rendre au travail.

b. \{Plusieurs / *Quelques\} genres de difficultés se présentent à nous.

Ces exemples montrent que les entités désignées par les noms taxinomiques ne peuvent se regrouper, sous la portée de quelques, qu'à certaines conditions. Elles opposent donc une résistance au mode de collectivisation imposé par quelques.

Ces dernières observations constituent des directions de recherche. Mais l'hypothèse de travail avancée au début de cet article est validée sur plusieurs sortes d'exemples : les pluriels (comme gouttes, cheveux, connaissances), les $\mathrm{N}$ intrinsèquement pluriels (manger quelques pâtes, avoir quelques rondeurs) et les entités ayant une structure interne homogène (d'extension temporelle comme instant, moment, ou spatiale comme geste, nuage). 
Ces faits peuvent être ramenés à une seule proposition représentable dans le cadre théorique de la grammaire cognitive : les deux déterminants profilent différemment la pluralité dénotée par le groupe nominal. Quelques $N$ dénote une collectivité constituée d'un nombre indéterminé d'éléments, alors que plusieurs $N$ dénote une sommation (indéterminée) de N. Nous pensons que cette différence de profilage a un impact sur des exemples que l'on aurait tendance, dans d'autres cadres théoriques, à considérer comme parfaitement synonymes :

\section{5. quelques murs / plusieurs murs}

A première vue, en effet, la commutation de quelques et de plusieurs dans 25 ne change pas grand-chose au sens du syntagme. Nous soutenons toutefois que les murs sont profilés de manière différente selon qu'ils sont déterminés par quelques ou plusieurs : quelques murs profile un collectif alors que plusieurs murs profile une pluralité.

Or, les exemples extraits de Frantext vont dans ce sens. Voici les deux occurrences de plusieurs murs :

26. a. Ce palais est habité en ce moment par un détachement de dames du harem qui ont quitté Fez la semaine dernière - et qui, bien entendu, ont été soigneusement séquestrées derrière plusieurs murs avant notre arrivée dans les jardins.

b. Il a pu voir, sur plusieurs murs, en venant à pied de son hôtel à lui, de l'avenue des ChampsElysées à la place Vendôme, l'affiche qui annonce le Colonel Chabert, et l'image du comédien célèbre l'obsède plus que jamais.

Sous la portée atomisante de plusieurs, les murs sont conçus comme distincts et ne peuvent renvoyer aux éléments d'une entité collective. Dans l'exemple 26a, nous avons ainsi affaire à pluralité d'enceintes. Quant aux murs de l'exemple 26b, ils appartiennent clairement à des immeubles différents.

Dans le contexte de quelques, en revanche, les choses se passent différemment :

27. a. Au bout de cette plaine, à environ deux lieues dans les terres, on aperçoit un mamelon qui porte quelques murs fortifiés sur sa cime, et qui protége de son ombre une bourgade en ruine : c'est là Argos.

b. Des bouquets d'eucalyptus de-ci, de-là, des groupes de pins parasols au-dessus de quelques murs écroulés, voilà toute la végétation que rencontrait le regard d'Alba Steno.

c. Derrière cette aile dégradée de l'ancien monastère, on voyait un monceau de ruines recouvertes à demi de végétations pariétaires, quelques murs encore debout, percés à jour, et de grandes fenêtres sans châssis par lesquelles on voyait le ciel.

Dans ces exemples, quelques murs est amené à désigner des ruines ou des vestiges, c'est-à-dire des entités collectives constituées de murs. Si plusieurs n'est pas impossible dans ces exemples, on voit bien que le profilage qu'il impose n'est pas tout à fait adapté aux exigences d'un contexte qui cherche plutôt à mettre en relief un collectif de murs, et non une pluralité de murs distincts.

\section{Références bibliographiques}

Asnès, M. (2006). «Prédication télique et atélique : résolution d'un paradoxe». In Indéfini et prédication, éds. F. Corblin, S. Ferrando et L. Kupferman, pp. 337-346. PUPS : Paris.

Bacha, J. (1997). «Entre le plus et le moins : l'ambivalence du déterminant plusieurs », Langue Française, 116, pp. 49-60.

Bosveld-de Smet, L. (2000). « Du pluriel au massif», Verbum, XXII, pp. 363-378.

Corblin, F. (2002). Représentation du discours et sémantique formelle. PUF : Paris.

Corblin, F. (2006). « Défini et prédication partielle ». In La relation partie-tout, éds. G. Kleiber, C. Schnedecker et A. Theissen, pp. 465-481. Peeters : Louvain.

Culioli, A. (1983). «A propos de quelque ». In Linguistique, énonciation. Aspects et détermination, éds. S. Fisher et J.-J. Franckel, pp. 21-29. Editions de l'EHESS : Paris. 
Dobrovie-Sorin, C., et Beyssade, C. (2005). Définir les indéfinis. CNRS Editions : Paris.

Ducrot, O. (1980). Les échelles argumentatives. Minuit : Paris.

Flaux, N. (1997). «Les déterminants et le nombre ». In Entre général et particulier : les déterminants, éds. N. Flaux, D. Van de Velde et W. Mulder, pp. 15-82. Artois Presses Université : Arras.

Flaux, N. (1999). «A propos des noms collectifs », Revue de linguistique romane, 63, pp. 471-502.

Flaux, N., et Van de Velde, D. (2000). Les noms en français : esquisse de classement. Ophrys : Paris.

Gaatone, D. (1991). «Les déterminants de la quantité peu élevée en français. Remarques sur les emplois de quelques et plusieurs », Revue romane, 21, pp. 3-13.

Gaatone, D. (2006). «La problématique des notions d' "indéfinitude" et de "prédication" à travers la comparaison de quelques et de plusieurs ». In Indéfini et prédication, éds. F. Corblin, S. Ferrando et L. Kupferman, pp. 25-35. PUPS : Paris.

Gondret, P. (1976). «"Quelques", "plusieurs", "certains", "divers" : étude sémantique », Le Français Moderne, 44, pp. 143-152.

Jayez, J. (2005). « Combien est-on à plusieurs? ». Présenté au colloque Indefinites and Weak Quantifiers, Bruxelles.

Kleiber, G. (1997). «Massif / comptable et partie / tout », Verbum, 3, pp. 321-327.

Lammert, M. (2006a). Sémantique et cognition : les noms collectifs, Thèse de doctorat, UFR des Lettres, Université Marc Bloch. Strasbourg.

Lammert, M. (2006b). « Marqueurs de partition : outils métalinguistiques dans l'analyse des noms collectifs ». In $L a$ relation partie-tout, éds. G. Kleiber, C. Schnedecker et A. Theissen. Peeters: Louvain.

Landman, F. (1989). « Groups I », Linguistics \& Philosophy, 12, pp. 559-605.

Langacker, R. W. (1987). Foundations of Cognitive Grammar : Theoretical Prerequisites. Vol. 1. Stanford University Press : Stanford.

Langacker, R. W. (1991). Foundations of Cognitive Grammar : Descriptive Application. Vol. 2. Stanford University Press : Stanford.

Lebart, L., et Salem, A. (1994). Statistique textuelle. Dunod : Paris.

Lecolle, M. (1998). « Noms collectifs et méronymie », Cahiers de grammaire, 23, pp. 41-65.

Leeman, D. (2004). Les déterminants du nom en français. Syntaxe et sémantique. PUF : Paris.

Link, G. (1983). «The Logical Analysis of Plurals and Mass Terms: A Lattice-Theoretical Approach ». In Meaning, Use, and Interpretation of Language, éds. R. Bäuerle, C. Schwarze et A.v. Stechow : Berlin.

Muller, C. (1973). Principes et méthodes de statistique lexicale. Champion : Paris.

Nef, F. (1980). «Les verbes aspectuels du français: remarques sémantiques et esquisse d'un traitement formel ». Semantikos, vol. $4, \mathrm{n}^{\circ} 1$, pp. 11-46.

Paillard, D. (2006). "Quelque N / Quelques N ». In Indéfini et prédication, éds. F. Corblin, S. Ferrando et L. Kupferman, pp. 417-428. PUPS : Paris.

Spector, B. (2006). Aspects de la pragmatique des opérateurs logiques, Thèse de doctorat, Sciences du Langage, Université Paris 7. Paris.

\footnotetext{
${ }^{1}$ Je tiens à remercier Michelle Lecolle pour sa relecture et ses remarques, Sarah Leroy pour ses encouragements, Sylvain Loiseau pour son aide décisive, et enfin, Marie-Laurence Knittel pour m'avoir lancé sur cette problématique.

${ }^{2}$ Gondret (1976), Gaatone (1991) et Flaux (1997).

${ }^{3}$ Le nombre important de réponses et la difficulté d'utilisation des grammaires dans Frantext nous ont amené à ne pas faire de requête plus élaborée qui permettrait d'extraire des groupes nominaux plus complexes.
} 
${ }^{4}$ Le chiffre (colonne freq) indique le nombre d'occurrences de chaque substantif (colonne lemme) lorsqu'il suit le déterminant. Les items sont classés par ordre de fréquence décroissante.

${ }^{5}$ Cf. en particulier Muller (1973) ou Lebart \& Salem (1994). La loi hypergéométrique consiste à générer tous les sous-corpus possibles de même taille que le sous-corpus observé, à partir du corpus entier. Pour tous les corpus possibles, on regarde combien d'occurrences il y a de la forme dont on veut les spécificités. Par exemple, si 40 souscorpus sur 1000 sous-corpus possibles ont 20 occurrences, la probabilité de trouver 20 occurrences est de 40 pour 1000 .

${ }^{6}$ Dans ce tableau comme ceux qui suivent, les items sont classés par spécificité décroissante à l'exception de ceux qui ont un taux de 1, qui sont classés par ordre alphabétique.

${ }^{7}$ A titre d'exemple, en voici une occurrence dans Frantext : «Le défaut général est la longueur. De là résultent des répétitions d'idées. Il faut supprimer plusieurs vers et faire quelques-uns [sic]. » (Flaubert - Correspondance).

${ }^{8}$ Pour faciliter l'accès aux fréquences des occurrences dans les sous-corpus $\mathrm{Q}$ et $\mathrm{P}$, les numéros de rang des noms concernés sont donnés entre parenthèses à la fin des exemples.

${ }^{9}$ En essayant d'étendre au pluriel quelques les propositions de Culioli (1984) sur le singulier quelque, Paillard (2006) avance des éléments supplémentaires en faveur de cette hypothèse.

${ }^{10}$ Afin d'éviter les malentendus, précisons une fois pour toutes que quelques cheveux désigne une petite chevelure dans ce contexte. Ce n'est évidemment pas le cas pour toutes les occurrences de quelques cheveux : "il a quelques cheveux sur son veston ». Cette remarque est valable pour tous les autres exemples traités ici.

${ }^{11}$ Dans Frantext, toutes les unités monétaires constituent une spécificité positive de quelques. Par ordre de spécificité décroissante, on a successivement: sous (rang 2), louis (49), francs (51), écus (57), centimes (138), liards (189), pistoles (345), sols (388), napoléons (396), ducats (403), piastres (408) et enfin dollars (476). Frantext n'étant toujours pas passé à l'euro, on n'y trouve aucune occurrence de quelques euros, et a fortiori, de plusieurs euros.

${ }^{12}$ Cf. Link (1983), Landman (1989), Corblin (2002), Corblin (2006).

${ }^{13}$ A l'exception de $14 \mathrm{c}$.

${ }^{14}$ Notons toutefois que l'homogénéité interne des noms collectifs est relativisée par Lammert (2006a) et (2006b).

${ }^{15}$ Sur les différentes relations partie / tout et surtout, une tentative intéressante d'isoler les propriétés de la relation membre / collection, nous renvoyons à Lecolle (1998).

${ }^{16}$ Nous reviendrons rapidement sur la question de la lecture taxinomique que l'on trouve souvent associée à plusieurs en opposition à une lecture collective avec quelques (section 5). On ne peut toutefois s'empêcher de noter ici le rapprochement possible avec la pluralisation des massifs, qui donne elle aussi une lecture taxinomique.

${ }^{17}$ Rang 10 et $14 \mathrm{du}$ Tableau 2. A noter, les 25 occurrences de la forme latine instans dans Frantext catégorisé, au rang 98 du même tableau.

${ }^{18}$ Cf. Nef (1980).

${ }^{19}$ Ce contexte permet en outre d'opposer plusieurs aux numéraux auxquels on a pourtant tendance à l'associer : Il a terminé sa soupe en \{quelques / trois / *plusieurs\} minutes.

${ }^{20}$ Ce point est à rapprocher de ce que l'on observe dans l'exemple $17 \mathrm{~b}$ (Ce soir, on mange plusieurs pâtes) où la lecture taxinomique est la seule possible. 